\title{
DEPTH-AVERAGED MODEL INCLUDING VELOCITY DEFORMATION FOR UNSTEADY OPEN CHANNEL FLOWS
}

\author{
Manoj LANGHI ${ }^{1}$, Takashi HOSODA ${ }^{2}$ and Subhasish DEY ${ }^{3}$ \\ ${ }^{1}$ Member of JSCE, Ph. D. student, Dept. of Urban Management, Graduate School of Engineering, Kyoto University \\ (C1-3 Kyoto-Daigaku-Katsura, Nishikyo-ku, Kyoto 615-8540, Japan) \\ ${ }^{2}$ Member of JSCE, Professor, Dept. of Urban Management, Kyoto University (C1-3 Kyoto-Daigaku-Katsura, \\ Nishikyo-ku, Kyoto 615-8540, Japan) \\ 3 Professor, Dept. of Civil Engineering, Indian Institute of Technology (Kharagpur 721-302, W. B., India)
}

\begin{abstract}
To examine the characteristics of unsteady open channel flow, one-dimensional depth-averaged velocity deformation model is developed. The fundamental form of streamwise velocity in a power series of depth is initially assumed, and the formula for friction velocity is derived. The coefficients of the power series are later evaluated by using the unsteady equation of motion. The applicability of the model is tested using experimental data. The numerical model showed reasonably good agreement with the experimental data. A new approach to reproduce the velocity deformation in the vicinity of free surface using the comparison of velocity distribution of numerical model and uniform velocity distribution of Engelund model is also presented.
\end{abstract}

Key Words : unsteady, open channel flow, hydrodynamic, velocity deformation

\section{INTRODUCTION}

Unsteady effect in the rivers influences the flow during floods causing the loop-rating curve for discharge against flow depth. The behavior of the flow changes with the flood period during the rising and falling stages. Investigation of velocity distribution is therefore one of the most important aspects to study the hydrodynamic characteristics of rivers during floods. The simple depth-averaged model is a powerful tool to characterize the flow in the rivers due to its small computational loads. The key idea is to assume the velocity distribution throughout the depth and to estimate the bed shear stress by the momentum equation.

Many researchers accurately measured the characteristics of unsteady flows despite of the difficulty in measurements near the free surface, of which Nezu et. al. (1997) conducted turbulence measurements over a smooth bed, while Song and Graf (1996) performed the measurements over a rough bed.

Numerical simulation of flood flows was carried out by Nezu et. al. (2006), Hosoda et. al. (2010) and others. Onda et. al. (2003) developed a simple model for velocity distribution in steady state by incorporating the accelerated and decelerated effect of the flow.

Both Engelund's model (1974) and the model deduced by Onda et. al. (2003) did not consider the unsteady effect of the flow. Thus, to study the characteristics of a flood flow, we propose a simple depth-averaged model incorporating an unsteadiness parameter. To check the applicability of the model for unsteady flows over smooth and rough beds, the experimental data of Song and Graf (1996) and Nezu et. al. (1997) are used.

\section{MODEL FORMULATION}

(1) Distribution of streamwise velocity over entire depth

The vertical distribution of streamwise velocity for Engelund (1974) model is described by the equation of motion.

$$
\frac{1}{\rho} \frac{\partial \tau}{\partial y}=-g \sin \theta
$$

where $\tau$ is bed shear stress and $\theta$ is bed slope. 
The model adopted the assumptions of a constant eddy viscosity $\varepsilon$ in a steady uniform flow for a wide channel.

$$
\frac{\tau}{\rho}=\varepsilon \frac{d u}{d y} ; \quad \varepsilon=\beta u_{*} h, \quad \beta=0.077
$$

To simulate the unsteady open channel flows, we consider the unsteady effect of the flow in the model for the evaluation of streamwise velocity in vertical direction. The distribution of streamwise velocity over entire depth is expressed by a power law as

$$
\frac{u}{U}=u_{0}+u_{1} \eta+u_{2} \eta^{2}+u_{3} \eta^{3}+u_{4} \eta^{4}
$$

where, $U$ is the average velocity and $\eta=y / h$. By substituting the above equation into the continuity equation (Eq. 4).

$$
\frac{\partial u}{\partial x}+\frac{\partial v}{\partial y}=0
$$

and integrating Eq. (4) from the bottom to the water surface, the vertical velocity $v$ is obtained as,

$$
\begin{aligned}
v= & -h \frac{\partial U}{\partial x}\left(\sum_{i=0}^{4} \frac{1}{i+1} u_{i} \eta^{i+1}\right) \\
& +U \frac{\partial h}{\partial x}\left(\sum_{i=1}^{4} \frac{i}{i+1} u_{i} \eta^{i+1}\right)
\end{aligned}
$$

The hydrostatic pressure $p$ is expressed as

$$
\frac{1}{\rho} \frac{\partial p}{\partial x}=g \cos \theta \frac{\partial h}{\partial x}
$$

Substituting Eq. (2), (3), (5) and (6) into equation of motion in $x$-direction,

$$
\frac{\partial u}{\partial t}+u \frac{\partial u}{\partial x}+v \frac{\partial u}{\partial y}=g \sin \theta-\frac{1}{\rho} \frac{\partial p}{\partial x}+\frac{\partial}{\partial y}\left(\frac{\tau_{x y}}{\rho}\right)
$$

and rearranging the terms to the fourth power of $\eta$, the following equation is obtained:

$\sum_{i=0}^{4}\left(\frac{\partial U}{\partial t} u_{i}-i \frac{U}{h} \frac{\partial h}{\partial t} u_{i}\right) \eta^{i}+U \frac{\partial U}{\partial x}\left[u_{0}^{2}+u_{0} u_{1} \eta+\frac{1}{2}\right.$

$u_{1}^{2} \eta^{2}-\left(u_{0} u_{3}-\frac{2}{3} u_{1} u_{2}\right) \eta^{3}+\left(\frac{1}{3} u_{2}^{2}-2 u_{0} u_{4}+\frac{1}{4} u_{1} u_{3}\right)$

$\left.\eta^{4}\right]-\frac{U^{2}}{h} \frac{\partial h}{\partial x}\left[u_{0} u_{1} \eta+\left(\frac{1}{2} u_{1}^{2}+2 u_{0} u_{2}\right) \eta^{2}+\left(3 u_{0} u_{3}+\right.\right.$

$\left.\left.\frac{4}{3} u_{1} u_{2}\right) \eta^{3}+\left(\frac{2}{3} u_{2}^{2}+4 u_{0} u_{4}+\frac{7}{4} u_{1} u_{3}\right) \eta^{4}\right]-g \sin \theta$

$+g \cos \theta \frac{\partial h}{\partial x}-\beta h u_{*} \frac{U}{h^{2}}\left(2 u_{2}+6 u_{3} \eta+12 u_{4} \eta^{2}\right)=0$

The coefficients of power series (Eq. 3) are evaluated by considering each term of Eq. (8) in different order of $\eta$ to zero. This procedure is used to calculate the coefficients $u_{2}$ and $u_{3}$ as,

$$
\begin{gathered}
u_{2}=\frac{h}{2 \beta u_{*} U}\left(u_{0} \frac{\partial U}{\partial t}+u_{0}^{2} U \frac{\partial U}{\partial x}\right. \\
\left.\quad-g \sin \theta+g \cos \theta \frac{\partial h}{\partial x}\right) \\
u_{3}=\frac{h u_{1}}{6 \beta u_{*} U}\left(\frac{\partial U}{\partial t}-\frac{U}{h} \frac{\partial h}{\partial t}+u_{0} U \frac{\partial U}{\partial x}-u_{0} \frac{U^{2}}{h} \frac{\partial h}{\partial x}\right)
\end{gathered}
$$

However, $u_{0}$ is defined by the expression of bottom velocity,

$$
u_{0}=r_{*} \frac{u_{*}}{U}
$$

and $u_{1}$ is described by the definition of the bed shear stress as

$$
u_{1}=\frac{u_{*}}{\beta U}
$$

Considering zero shear stress at the water surface, the coefficient $u_{4}$ is evaluated as

$$
u_{4}=-\frac{1}{4}\left(u_{1}+2 u_{2}+3 u_{3}\right)
$$

(2) Calculation procedure for vertical distribution of streamwise velocity

By definition

$$
U h=\int_{0}^{h} u d y
$$

Eq. (3) is integrated in the following form,

$$
u_{0}+\frac{1}{2} u_{1}+\frac{1}{3} u_{2}+\frac{1}{4} u_{3}+\frac{1}{5} u_{4}=1
$$

Substituting all the expressions of coefficients in Eq. (15) and then after simplification, the equation for friction velocity is obtained as follows:

$$
\begin{aligned}
u_{*}^{2}= & c_{f} u^{2}\left(1+A_{1} \frac{\partial U}{\partial t}+A_{2} \frac{\partial h}{\partial t}\right. \\
& \left.+B_{1} \frac{\partial U}{\partial x}+B_{2} \frac{\partial h}{\partial x}+C_{1}-C_{2}\right)
\end{aligned}
$$

where,

$$
\begin{aligned}
A_{1}= & \frac{1}{\beta} \frac{h}{U^{2}}\left(\frac{7 r_{*}}{30}+\frac{1}{30 \beta}\right)\left[-1-\frac{1}{60 \beta^{2}} \frac{1}{U} \frac{\partial h}{\partial t}\right. \\
& \left.+\frac{1}{4 \beta} \frac{h}{U^{2}}\left(\frac{7 r_{*}}{30}+\frac{1}{30 \beta}\right) \frac{\partial U}{\partial t}\right] \\
A_{2}= & \frac{1}{30 \beta^{2}} \frac{1}{U}\left(1+\frac{1}{120 \beta^{2}} \frac{1}{U} \frac{\partial h}{\partial t}\right)
\end{aligned}
$$




$$
\begin{aligned}
& B_{1}=\frac{7 r_{*}}{60 \beta^{2}} \frac{h^{2}}{U^{3}}\left(\frac{7 r_{*}}{30}+\frac{1}{30 \beta}\right) \times \\
& B_{2}=-\frac{7 r_{*}}{1800 \beta^{3}} \frac{h}{U^{2}}\left(g \sin \theta-g \cos \theta \frac{\partial h}{\partial x}\right) \\
& C_{1}=\frac{7}{10 \beta} \frac{h}{U^{2}}\left(\frac{r_{*}}{3}+\frac{3}{20 \beta}\right)\left(g \sin \theta-g \cos \theta \frac{\partial h}{\partial x}\right) \\
& C_{2}=\left[-1+\frac{1}{2 \beta} \frac{h}{U^{2}} \frac{\partial U}{\partial t}\left(\frac{7 r_{*}}{30}+\frac{1}{30 \beta}\right)\right. \\
& \left.\quad-\frac{1}{60 \beta^{2}} \frac{1}{U} \frac{\partial h}{\partial t}\right] \times\left(1+A_{1} \frac{\partial U}{\partial t}+A_{2} \frac{\partial h}{\partial t}\right. \\
& \left.\quad+2 B_{1} \frac{\partial U}{\partial x}+2 B_{2} \frac{\partial h}{\partial x}+2 C_{1}\right)^{0.5} \\
& c_{f}=\left\{2 \left[\begin{array}{c}
r_{*}+\frac{9}{20 \beta}-\frac{r_{*}}{60 \beta^{2}} \frac{\partial h}{\partial x} \\
\left.\left.\frac{h}{U}\left(\frac{7 r_{*}}{30}+\frac{1}{30 \beta}\right) \frac{\partial U}{\partial x}\right]^{2}\right\}^{-1}
\end{array}\right.\right.
\end{aligned}
$$

The solution of Eq. 16 is used to determine the values of all other coefficients of velocity. The coefficient $r_{*}$ is decided in such a way that the base flow discharge is obtained as a initial condition. During the simulation, the discharge hydrograph at the upstream end and constant depth at the downstream end are used as an initial and boundary condition. The depth-averaged equation is later solved by including the effect of vertical distribution of streamwise velocity in the momentum equation.

$$
\begin{gathered}
\frac{\partial U h}{\partial t}+\frac{\partial}{\partial x}\left(g \frac{h^{2}}{2}\right)+\left[u_{0}^{2}+u_{0} u_{1}+\frac{1}{3}\left(u_{1}^{2}+2 u_{0} u_{2}\right)\right. \\
+\frac{1}{4}\left(2 u_{0} u_{3}+2 u_{1} u_{2}\right)+\frac{1}{5}\left(u_{2}^{2}+2 u_{0} u_{4}+2 u_{1} u_{3}\right) \\
\left.+\frac{1}{6}\left(2 u_{1} u_{4}+2 u_{2} u_{3}\right)\right] \frac{\partial}{\partial x}\left(U^{2} h\right)=g h \sin \theta-u_{*}^{2}
\end{gathered}
$$

\section{RESULTS}

To verify the applicability of the proposed numerical (Num) model, the experimental data of Song and Graf (1996) for rough bed case; and Nezu et. al. (1997) for smooth bed case (with high and low unsteadiness parameter) are used for the comparison. The hydraulic conditions considered during the simulation are tabulated in Table 1.
Table 1 Hydraulic conditions considered during simulation.

\begin{tabular}{|c|c|c|c|c|c|}
\hline Case & Slope & $T_{d}(\mathrm{sec})$ & $Q_{b}\left(\mathrm{~m}^{3} / \mathrm{s}\right)$ & $Q_{p}\left(\mathrm{~m}^{3} / \mathrm{s}\right)$ & $h_{b}(\mathrm{~m})$ \\
\hline \hline SG & 0.003 & 52 & 0.0585 & 0.0891 & 0.11 \\
\hline NZ1 & 0.00167 & 60 & 0.005 & 0.0154 & 0.0405 \\
\hline NZ2 & 0.00167 & 120 & 0.005 & 0.0161 & 0.041 \\
\hline
\end{tabular}

In Table $1, T_{d}$ is the duration from base flow to peak flow discharge; $Q_{b}$ is the base flow discharge; $Q_{p}$ is the peak flow discharge and $h_{b}$ is the base flow depth. For all cases $\Delta x=0.05$ and $\Delta t=0.01$.

\section{(1) Smooth bed case \\ a) Bed shear stress}

The friction velocity evaluated from Eq. 16 is used to determine the bed shear stress. This bed shear stress $\tau_{w}$ is then normalized by mean bed shear stress $\overline{\tau_{w}}$. The normalized bed shear stress against normalized time for cases NZ1 (high unsteadiness parameter) and NZ2 (low unsteadiness parameter) along with the experimental data are illustrated in Fig. 1. The depth hydrographs ( $\Delta h=h-h_{b}$ ) (depth vs. time) for these cases are also depicted in Fig. 1. As in experiments, the peak of normalized bed shear stress decreases with a decrease in unsteadiness parameter. It is also evident from Fig. 1 that during the passage of flood flows, the friction velocity is attained to its maximum value first and after the peak of depth appears.

\section{b) Velocity deformation}

In open channel flows, the vertical distribution of streamwise velocity deviates at the free surface due to attenuation of vertical turbulence intensity. In the present model, instead of vertical turbulence intensity the additional stresses which are formed due to the correlation of time-averaged vertical and streamwise velocity are incorporated. These additional stresses in Num model causes the vertical distribution of streamwise velocity to deviate from the uniform flow velocity distribution of Engelund model (EM) $U_{E M}$. This velocity deformation induced by unsteadiness and non-uniformity of the flow is depicted in Fig. 2. The temporal change of difference of non dimensional velocity at the surface is compared with the experimental result for both the cases, as shown in Fig. 3. Here, the $\prod$ value for steady flow in experimental case is selected as 0.1. Similar to the experiment, the distribution of normalized velocity attains a maximum value before the peak depth appears. It is also seen that the peak values of normalized velocity decreases with a decrease in unsteadiness parameter. 

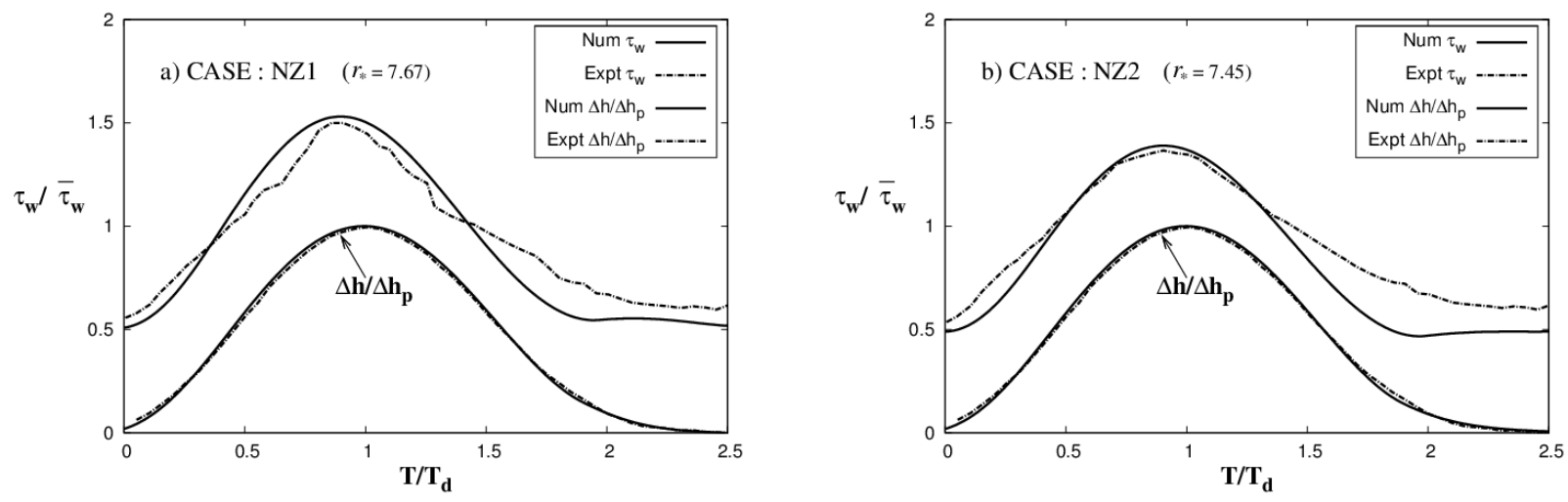

Fig. 1 Bed shear stress $\tau_{w}$ against time $t\left(=T / T_{d}\right)$ a) NZ1; b) NZ2 (at $x=7 \mathrm{~m}$ in $10 \mathrm{~m}$ long flume)
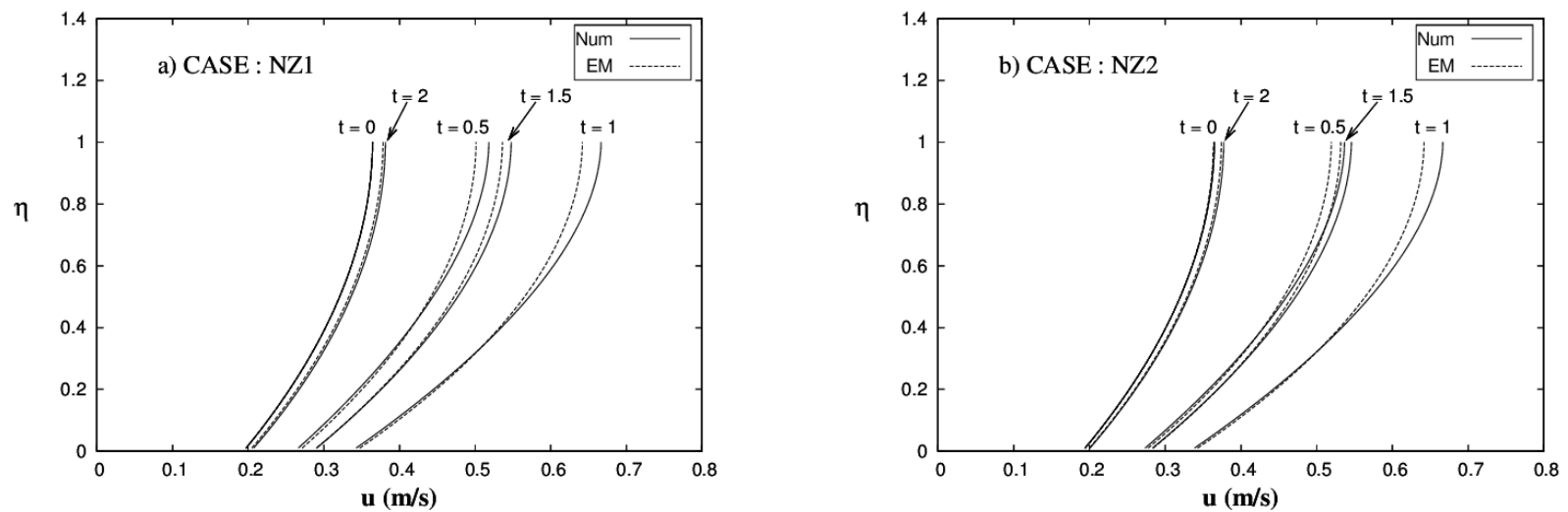

Fig. 2 The comparison of vertical distribution of streamwise velocity. a) NZ1; b) NZ2
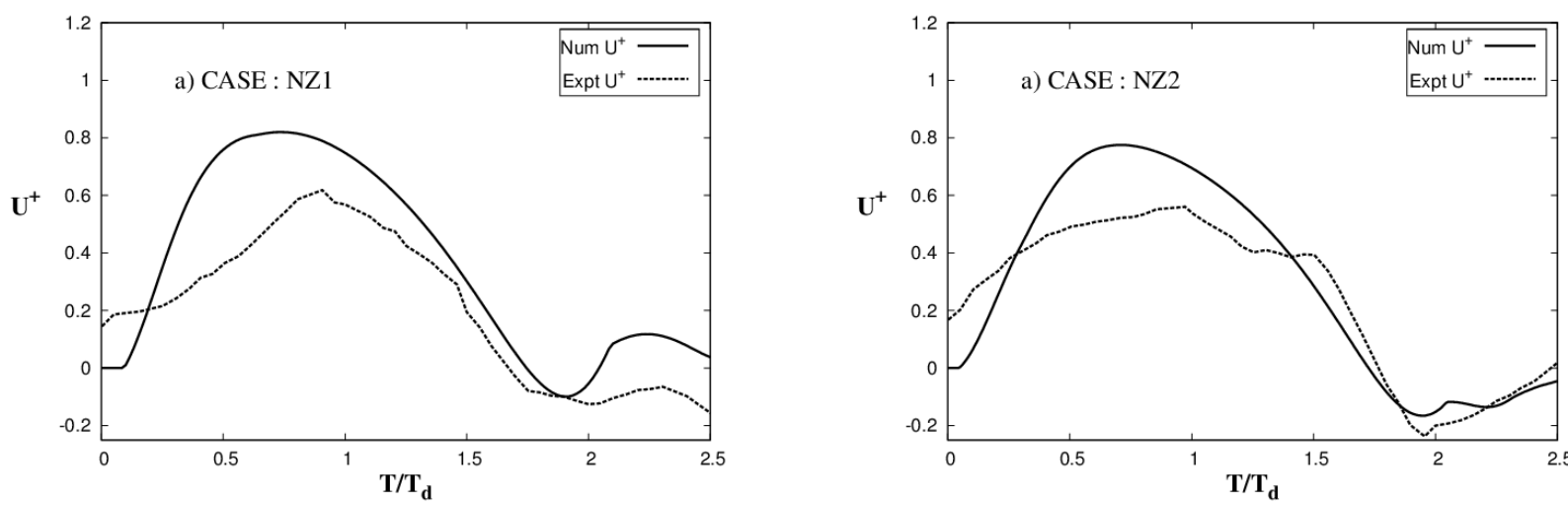

Fig. 3 Normalized Velocity $U^{+}=\left(U / u_{*}-U_{E M} / u_{*}\right)$ against time $t$ in the surface region . a) NZ1; b) NZ2

\section{(2)Rough bed case \\ a) Hydrograph}

Time variation of hydraulic parameters is depicted in Fig. 4 for rough bed case (SG). The comparison shows the hydrographs for Num model along with the experimental data of Song and Graf (1996). All hydrographs agree well with the experimental data. The consideration of power series for streamwise velocity over the entire depth is responsible for the higher peak velocity in Num model. During the passage of flow, maximum of friction velocity attained first and successively the peak of averaged velocity and discharge arrive. Finally, the maximum value of depth appears causing a loop-rating curve for depth against discharge.

\section{b) Time variation of streamwise velocity}

Time variation of streamwise velocities over entire depth is presented in Fig. 5. It is evident that near wall region, the computed velocity over estimates the experimental values but in the intermediate region the distribution is similar to the experiments. This is attributed to the friction near the bed to be less for Num model, causing higher velocity at the bottom compared to the experiment. Further up in the region, the computed velocity values are underestimated the experimental values 

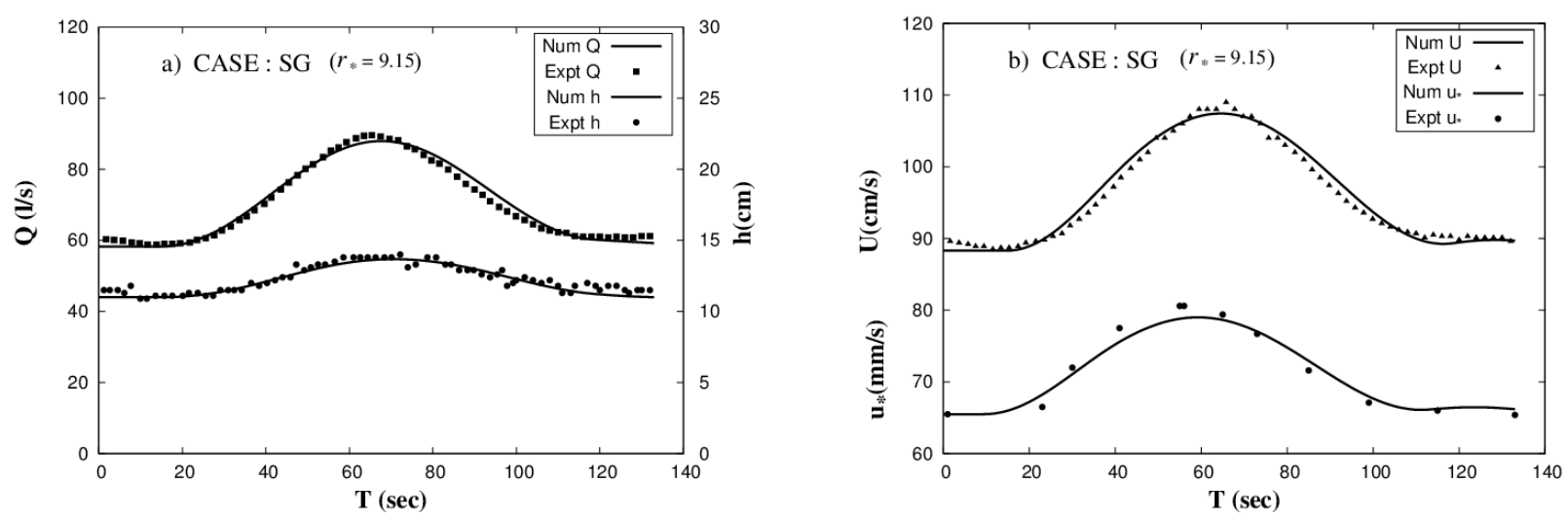

Fig. 4 Time variation of hydraulic parameters a) discharge and depth hydrographs; b) average and friction velocity hydrographs ( at $X=14.1 \mathrm{~m}$ in $16.8 \mathrm{~m}$ long flume)
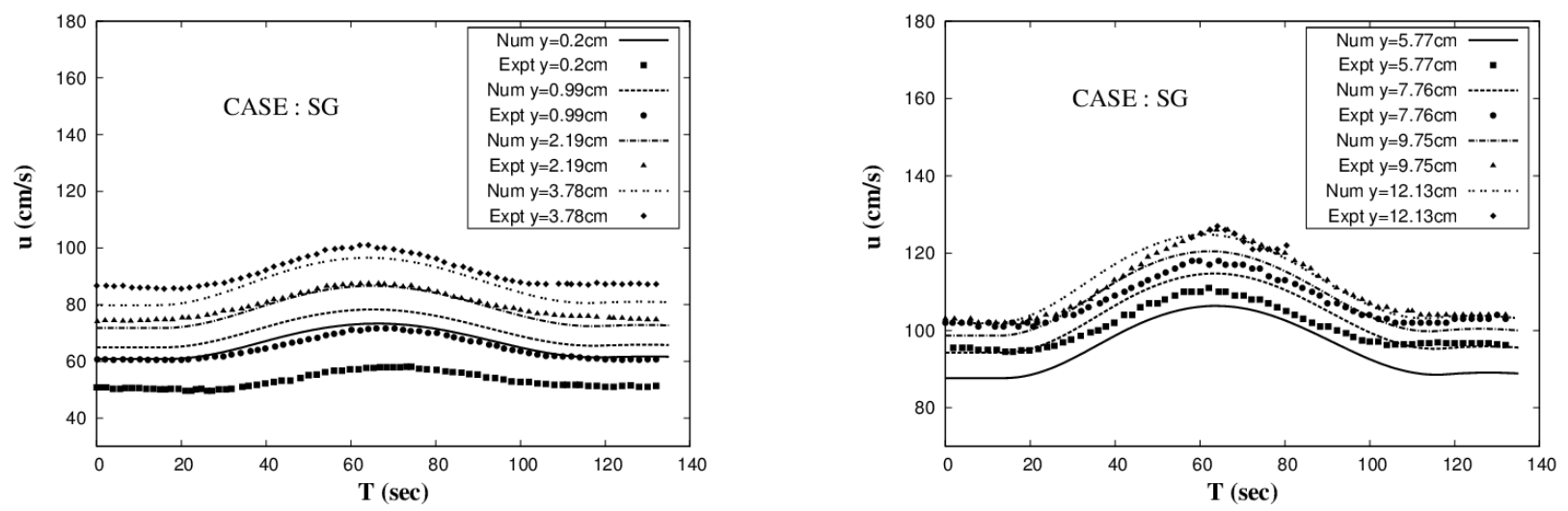

Fig. 5 Time variation of streamwise velocity over entire depth (Case : SG)

and at the free surface it again matches with the experimental data, indicating full development of the flow due to inclusion of an unsteadiness effect. As in the experiments, the velocity in the vicinity of water surface attains the peak values earlier than the velocity near the bottom.

\section{c) Vertical distribution of streamwise velocity}

The distributions of streamwise velocities over the entire depth for equivalent experimental depth are plotted in Fig. 6. The hydraulic parameters associated are tabulated in Table 2. Due to overestimation of the near wall velocity, the distribution does not show good agreement near the bottom for all the time. For the base flow, the velocity distribution is not as well developed as the experimental one. When the peak discharge approaches, the velocity distribution for Num model come closer to the experimental data. The vertical distribution of velocity during the rising stage shows the higher value compared to the falling stage, indicating that the sediment transport rate is higher in rising stage and lower in the falling stage. It is observed from the comparison that the secondary current causes the experimental velocity distribution to deviate in the vicinity of the free surface resulting
Table 2 The numerical and experimental hydraulic variables

\begin{tabular}{|c|c|c|c|c|}
\hline \multirow{2}{*}{$T(\mathrm{sec})$} & \multicolumn{2}{|c|}{ Experiment } & \multicolumn{2}{c|}{ Numerical (Num) } \\
\cline { 2 - 5 } & $h(\mathrm{~cm})$ & $u_{*}(\mathrm{~cm} / \mathrm{s})$ & $h(\mathrm{~cm})$ & $u_{*}(\mathrm{~cm} / \mathrm{s})$ \\
\hline \hline 1 & 11.3 & 6.55 & 11 & 6.55 \\
\hline 133 & 11.3 & 6.54 & 11 & 6.62 \\
\hline 41 & 12.1 & 7.75 & 12.17 & 7.56 \\
\hline 99 & 12.1 & 6.71 & 12.35 & 6.80 \\
\hline 55 & 13.2 & 8.06 & 13.19 & 7.88 \\
\hline 85 & 13.2 & 7.16 & 13.28 & 7.29 \\
\hline 65 & 13.7 & 7.94 & 13.61 & 7.86 \\
\hline 73 & 13.7 & 7.67 & 13.66 & 7.70 \\
\hline
\end{tabular}

in maximum velocity at a certain distance below the water surface. In case of Num model, the maximum velocity is obtained at the surface due to the additional stresses. These additional stresses supplied are induced by the fluid flow, but not by the turbulence. This stresses are responsible for full development of flow at the water surface. This is clearly observed in Fig. 7, when velocity distribution for two cases, rising $(T=35 \mathrm{sec})$ and 

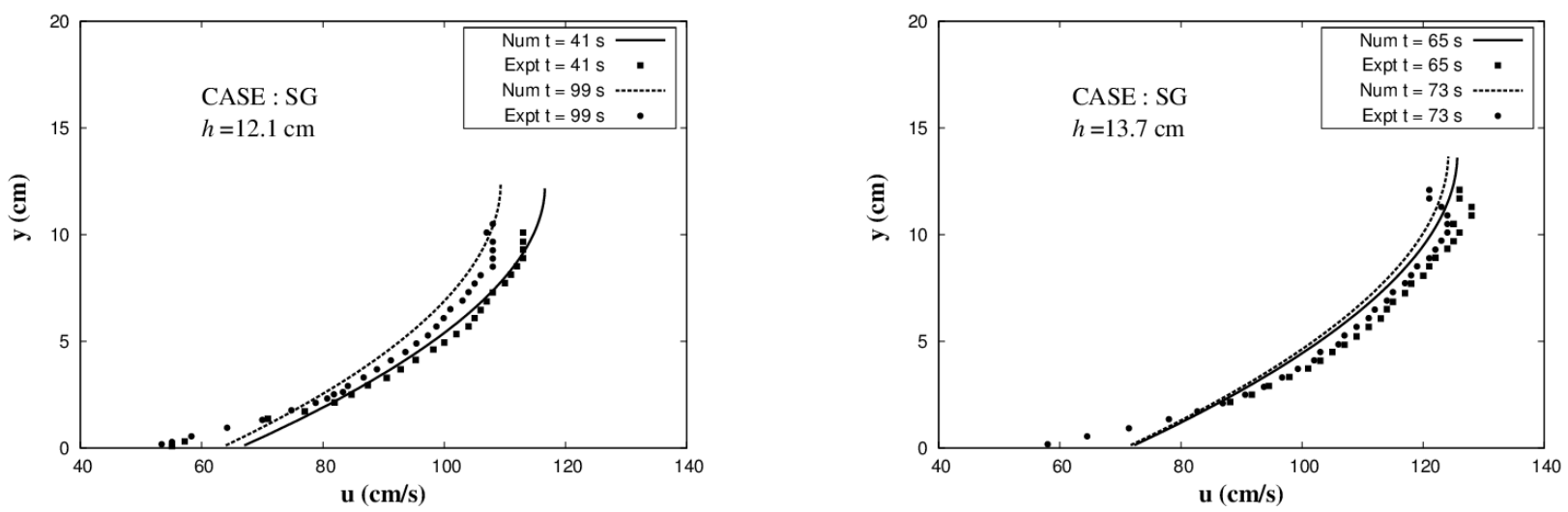

Fig. 6 The vertical distribution of streamwise velocity for equivalent depth during rising and falling stages
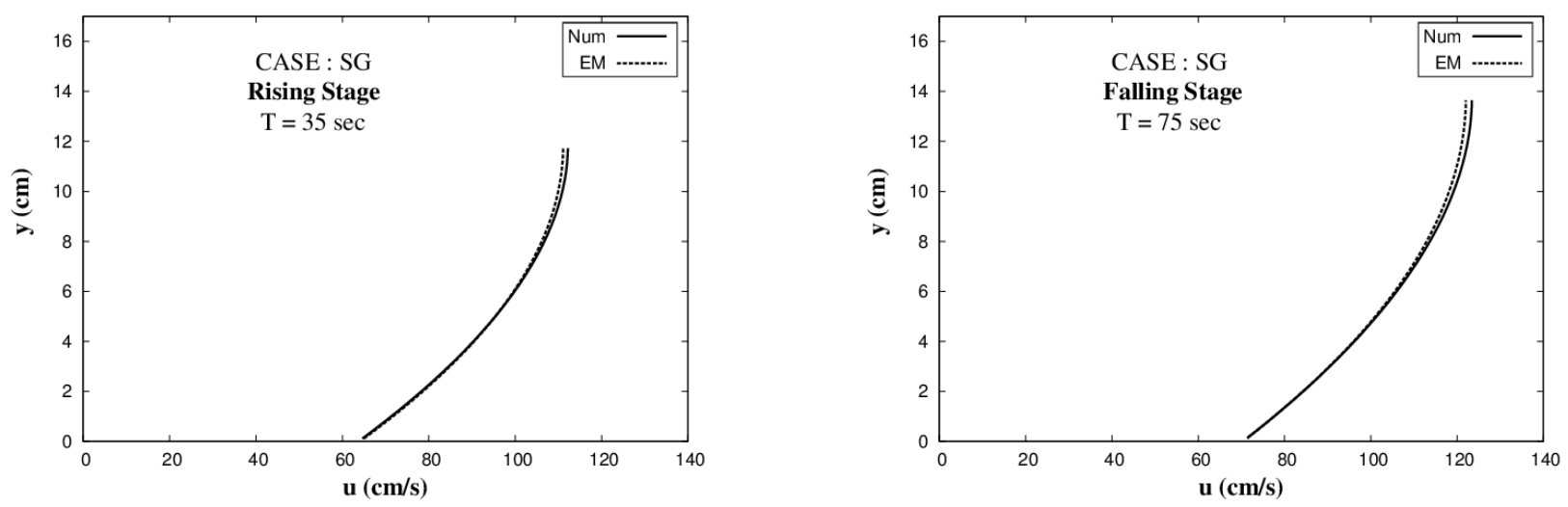

Fig. 7 The vertical distribution of streamwise velocity at a) $T=35 \mathrm{sec}$; and b) $T=75 \mathrm{sec}$.

falling $(T=75 \mathrm{sec})$, stage are plotted for both Num model and Engelund model. This deviation of velocity between Num model and Engelund model at free surface as pointed earlier, is caused by the unsteadiness and non uniformity of the flow.

\section{CONCLUSIONS}

The comparison of proposed depth-averaged velocity deformation model with the experimental data and computational result of EM model leads to the following conclusions:

The peak value of normalized bed shear stress decreases with a decrease in unsteadiness parameter.

Similar to the experiments, the velocity near the water surface attains the maximum value earlier than that near the wall.

As like the experiment, the streamwise velocity in the rising stage is larger than that in the falling stage for the equivalent water depth.

Additional shear stresses incorporated into the model are responsible for the deviation of velocity distribution of Num model from the uniform flow velocity distribution of Engelund model.

The difference of non dimensional velocity between Num model and Engelund model at the surface in the temporal distribution decreases at the peak with the decrease in unsteadiness parameter.

The results from the model agree well with the experimental data, suggesting that the model is well suited for the unsteady flow over the smooth and rough beds.

\section{REFERENCES}

1) Engelund, F.: Flow and bed topography in channel bends, $J$. Hydr. Div., ASCE, Vol. 100, pp.1631-1648, 1974.

2) Hosoda, T., Onda, S., Murakami, T., Iwata, M., Shibayama, Y., and Puay, H. T.: Flood flow simulation in the case of lack of both upstream and downstream boundary conditions, 17th IAHR-APD Congress and 7th IUWM Conference, Auckland, New Zealand, pp.1b014, 2010.

3) Nezu, I., Kadota, A., and Nakagawa, H.: Turbulent structure in unsteady depth varying open-channel flows, J. Hydr. Engg., ASCE, Vol. 123, pp.752-763, 1997.

4) Nezu, I., and Sanjou, M.: Numerical calculation of turbulence structure in depth varying unsteady open-channel flows, J. Hydr. Engg., ASCE, Vol. 132, pp.681-695, 2006.

5) Onda, S., Hosoda, T., and Kimura, I.: A simple model of a velocity distribution in accelerating/decelerating flows and its application to depth averaged flow model, Intern. Sympo. on Shallow flows, Delft, Netherlands, pp.637-644, 2004.

6) Song, T., and Graf, W. H.: Velocity and turbulence distribution in unsteady open channel flows, J. Hydr. Engg., ASCE, Vol. 122, pp.141-154,1996.

(Received September 30, 2011) 